\title{
Impact of a pressurised membrane: contact time
}

\author{
Loïc Tadrist ${ }^{\mathrm{a}, *}$, Baptiste Darbois Texier ${ }^{\mathrm{b}}$, François Lanzetta ${ }^{\mathrm{c}}$, Lounès Tadrist ${ }^{\mathrm{d}}$ \\ ${ }^{a}$ Aix-Marseille Université, CNRS, ISM, Marseille, France \\ ${ }^{b}$ Université Paris-Saclay, CNRS, FAST, Orsay, France \\ ${ }^{c}$ Université de Franche-Comté, CNRS, FEMTO-ST, Belfort, France \\ ${ }^{d}$ Aix-Marseille Université, CNRS, IUSTI, Marseille, France
}

\begin{abstract}
\end{abstract}
Keywords: pressurised membrane, impact, fluid-structure interaction, contact time

\section{Introduction}




\section{PARAMETERS}

$P \quad$ Absolute inner pressure

$P_{\text {atm }} \quad$ Atmospheric pressure $\quad 986 \mathrm{kPa}$

$\Delta P \quad$ Pressure variations during impact

V Gas volume

$T \quad$ Inner gas temperature

$R \quad$ Membrane radius

$h \quad$ Release height

$U_{0} \quad$ Impact speed

$g \quad$ Gravity acceleration

$t \quad$ Time

$x \quad$ Indentation

$x_{g} \quad$ Membrane center of mass

$t_{c} \quad$ Contact time

\section{MEMBrane PaRAmeters}

$\begin{array}{lll}R_{0} & \text { Radius (deflated) } & 8.2 \mathrm{~cm} \\ m & \text { Mass } & 179 \mathrm{~g} \\ E & \text { Young modulus } & 4.2 \mathrm{MPa} \\ e & \text { Thickness } & 2 \mathrm{~mm}\end{array}$

DiMENSIONLESS NUMBERS

$\gamma \quad$ Laplace coefficient

$\tilde{P} \quad$ Reduced pressure

$P_{E} \quad$ Presso-elastic number

$I_{P} \quad$ Presso-Inertial number

Table 1: Parameter and notations used in the paper. $(\dot{x})$ denotes the time derivative of $x$, index $\max$ denotes maximal value during impact. 
to increase head accelerations during ball-to-head impacts [3]. In rare situations, gas expansion is used to create impacts, for instance with a pneumatic jackhammer [4]. However, pressurised membranes are most often used to milden impacts: running shoes equipped with heel air pockets or foam (ensemble of small air pockets) protect from running impacts [5], inflatable bike helmet [6] or car air-bag [7] protect from traumatic brain injuries (TBI). During an impact, the membrane reaction force depends on inflation pressure which is, for instance, a major concern for airbagdeployment and gas dosage as it may cause facial or eye injuries $[8,9]$. The inertial forces created by pressurised membranes are proportional to the typical acceleration $U / t_{c}$ where $U$ is the impact speed and $t_{c}$ the contact time. Short contact time occurs for high pressure membranes and may be harmful. The relation between pressure, velocity and contact time is thus necessary to predict the danger of an impact of a pressurised membrane.

Contact time has already been observed on various objects. Contact time of inelastic balls has been investigated to understand granular material dynamics [10]. Impacting droplets were studied in order to reduce contact time to avoid possible pinning and keep surfaces clean $[11,12]$. Contact time of soap bubbles has also been measured and modelled [13]. Pressurised membranes differ from those system by having their mass in the membrane and gas as a spring.

The static deformation of pressurised structures have been studied on spherical and ellipsoidal models $[14,15]$, and is due to the competition between pressure and bending forces. An elastic shell deforms with an inverted cap instability [16], whereas the contact area of a pressurised membrane remains flat [17]. The dynamics of a membrane is mainly driven by gas pressure, membrane shape and bending rigidity[18-20]. During the impact, gas compression has been proposed to be adiabatic [18] or isothermal [21]. However, it has never been measured directly. Measurements of gas temperature, gas pressure and volume are performed simultaneously during the impact of a pressurized membrane.

In the following, we chose a spherical membrane (i.e. without bending rigidity) as a model experiment. The restoring force was only due to the combination of gas pressure and membrane shape. Differently from [21] where pressure elevation was not taken into account and from [18] where interaction between gas and membrane is not considered, we show that the main source of non-linearity arises from the membrane-gas interaction during the impact. Although impacts 
are usually characterised by contact time and coefficient of restitution, we mostly focused on the contact time of the impact.

In this paper, we first report measurements of the impact properties of pressurised membrane (contact time and coefficient of restitution) by systematically varying impact speed, inflation pressure and gas nature. We also describe the observed kinematics of the membrane during the impact. The second part of the paper is devoted to the gas dynamics during the impact and its strong interaction with the membrane elasticity. In a third part, we propose a model of the impact of a pressurised membrane. The impact time of a pressurised membrane is shown to be mainly dependent on two dimensionless numbers, the presso-inertial number and the presso-elastic number. A rationale is given for the dependency of the contact time with impact velocity arising from the non-linear equations of contact and gas dynamics of the pressurized membrane.

\section{Impacts on pressurised membranes : Experiments}

An impact is characterised by the contact time $t_{c}$, and the coefficient of restitution $\eta=\left|U_{\text {out }} / U_{0}\right|$. $\eta=1$ corresponds to a lossless impact where the outgoing speed is equal to the ingoing speed. We report here measurements of the impact properties of a pressurised membranes varying impact speed $U_{0}$, inflation pressure $P-P_{\text {atm }}$ and gas nature.

\subsection{Material and Methods}

Most studies of impacts on pressurized membranes have been carried out on sport balls [18, $21,22]$ for application to sports but also for supply and cost reasons. Tests were carried out on a set of 4 identical beach-volley balls (Decathlon KIPSTATM, BV100 fun). Those spherical membranes were chosen to be a reference experimental model. They have a minimal composition, they are formed of a single layer of rubber equipped with a valve for inflation, and they are soft enough to expand when inflated at usual inflation pressures and thus to exhibit a strong coupling between gas pressure and membrane elasticity. The membrane weighted $m=179 \mathrm{~g}$ and had a thickness $e=2.00 \pm 0.12 \mathrm{~mm}$. The Young modulus of the membrane was measured on a traction bench, $E=4.22 \pm 0.10 \mathrm{MPa}$. The deflated membrane radius was $R_{0}=8.2 \pm 0.1 \mathrm{~cm}$. All experiments 
were performed in the elastic range of the membrane (inflation between 5 and $35 \mathrm{kPa}$ ). The main membrane properties and notations are gathered in Table 1.

Membranes were inflated at four inflation pressures $P-P_{\mathrm{atm}}=0.1,0.15,0.25$ and $35 \mathrm{kPa}$ and their pressure cross checked with a precise manometer (MPX4250A, NXP). Three different gases were used for inflation, (i) air (diatomic gas with Laplace coefficient $\gamma=7 / 5$ ), (ii) pure Helium and (iii) pure Argon which are both monoatomic gases $(\gamma=5 / 3)$. The pressurised membranes were released at heights (top of the membrane, $h+2 R$ ) $0.5,0.75,0.875,1,1.25,1.35,1.5,1.75,1.85$ and $2 \mathrm{~m}$ with no velocity. The membranes impacted on a massive and non deformable marble stone, figure 1. Impacts were recorded thanks to a high-speed camera (Photron Miro 110) at 5000 frames per second.

Impact velocities recorded in the experiment varied from 1 to $6 \mathrm{~m} / \mathrm{s}$.Positions were determined by tracking the circular shape on each frame using Matlab ${ }^{\circledR} 2015 \mathrm{~b}$ algorithm. The algorithm works as follows, (i) first the scale in pixels per $\mathrm{mm}$ is defined and an approximate membrane radius is given after conversion in pixels. (ii) Second, the $\mathrm{i}^{\text {th }}$ image is loaded. (iii) Third, the membrane location is found using imfindcircle Matlab ${ }^{\circledR}$ function based on the circular Hough transform. This function outputs the radius and center of the circular shape of the pressurized spherical membrane. (iv) Four, the ground position is detected automatically using the Hough transform for lines. Steps (ii) to (iv) are repeated for all images of the high speed film. With this algorithm, the center of the circle has been measured precisely even when the membrane was deformed, see figure $1 \mathrm{~b}$. The algorithm results, ground position, the positions of the center of the circular shape of the membrane and its radius, were post treated. Contact occurred when the distance between the center of the circle and the marble is less than the membrane radius $R$. Consequently, contact time $t_{c}$ was defined precisely by fitting linearly the circle center-ground distance $v s$ time before and after impact. The absolute value of the slopes of those fits were used to determine the impact speed $U_{0}$ and the outgoing speed $U_{\text {out }}$. From these measurements, the coefficient of restitution was computed as $\eta=\left|U_{\text {out }} / U_{0}\right|$. 
a.

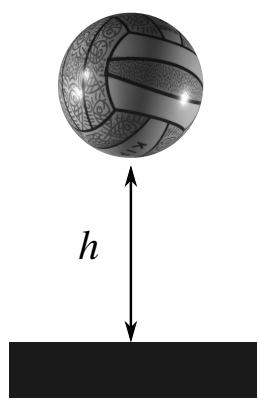

b.

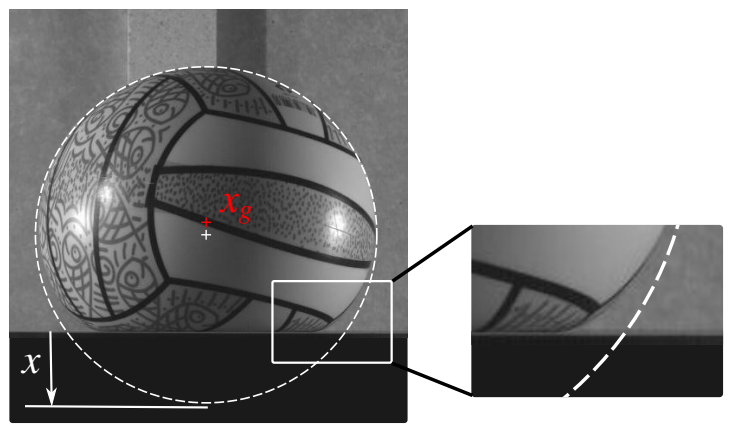

c.

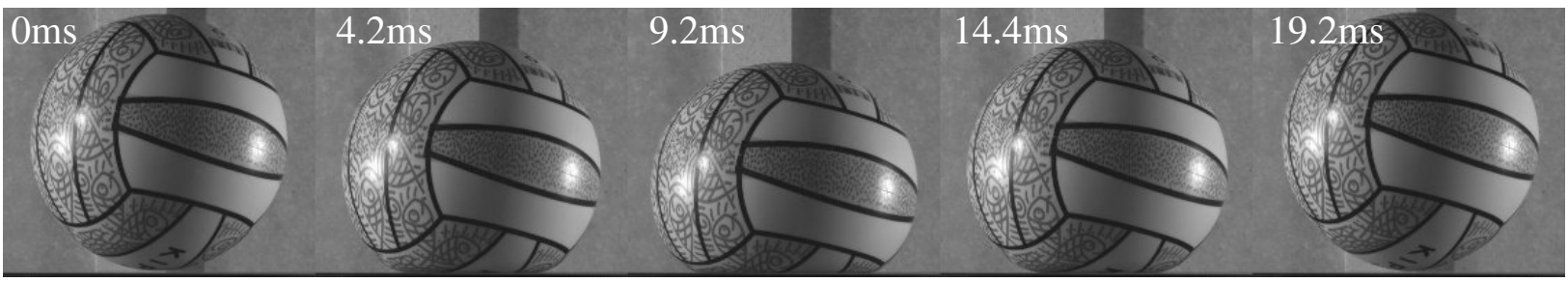

Figure 1: a. View of the experiment. The pressurised membrane is released without velocity from a height $h$. (1) Flicker-free lighting. (2) high speed camera. b. Definition of indentation $x$ and membrane center of mass $g$ during impact. Zoom on membrane oval distortion. c. Chrono-photography of an impact event $(h=1.85 \mathrm{~m}$ and $\left.P-P_{\text {atm }}=15 \mathrm{kPa}\right)$. 

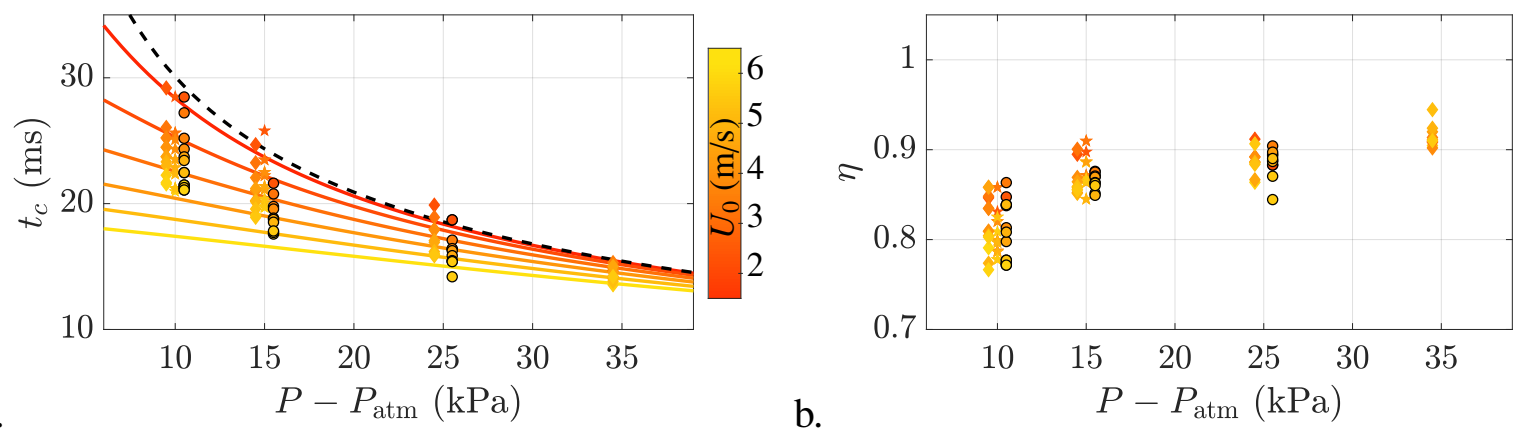

Figure 2: a. Contact time, $t_{c}$ as a function of inflation pressure $P-P_{\mathrm{atm}}$. Coloured lines are the non-dissipative model prediction for contact time, Eq. (15), for different impact speeds. The speed color map will be conserved for all the figures. Dashed line correspond to the linear model independent of impact speed $U_{0}$. b. Coefficient of restitution $\eta$ as a function of inflation pressure $P-P_{\mathrm{atm}}$. Inflation gas: Argon, $\star$ Helium and $\circ$ Air.

\subsection{Evolution of contact time as a function of inflation pressure, impact speed and gas nature}

The contact time $t_{c}$ is reported in figure $2 \mathrm{a}$ as a function of inflation pressure $P-P_{\mathrm{atm}}$ for different impact velocities. Impact velocities are colour-coded using the color scale of figure 1a. Contact time decreases with inflation pressure for a fixed impact speed. The spreading of impact times at a fixed inflation pressure is reduced when inflation pressure is increased.

For information, the coefficient of restitution $\eta=\left|U_{\text {out }} / U_{0}\right|$ is plotted in figure $2 \mathrm{~b}$ as a function of inflation pressure $P-P_{\text {atm }}$ for different impact velocities. The coefficient of restitution increases slightly with inflation pressure and decreases with impact speed.

\subsection{Kinematics of an impact of a spherical pressurised membrane}

During an impact onto the marble considered as a target of infinite mass and stiffness compared to the membrane, the spherical pressurised membrane may be described by the position of its center of mass $x_{g}$ or the indentation $x$, see figure $1 \mathrm{~b}$. The shape of the membrane during the impact is an indented spherical cap [18], which radius may slightly increase during the impact. The part of the membrane in contact with the ground is flattened and no ripples are observed. The volume, $V$, of a spherical cap and the flat area in contact with the ground, $A$, of a sphere of radius $R$ indented 
by a length $x$ reads,

$$
V(x)=\frac{\pi}{3}\left(4 R^{3}+x^{3}-3 R x^{2}\right) \quad \text { and } \quad A(x)=2 \pi R x\left(1-\frac{x}{2 R}\right) .
$$

The actual shape of the indented membrane differs from a pure sphere because of oval distortion, see zoom in figure $1 \mathrm{~b}$. This effect is taken into account in our model applying a correction to the actual surface in contact with the ground, $A_{\text {contact }}=\beta A$ with $\beta<1$. The effect of oval distortion on the volume of the spherical cap remains negligible.

The deformation of the ball moves its center of mass which is no longer located at the center of the spherical shape, see figure $1 \mathrm{~b}$. When the ball gets in contact with the ground, $x=0\left(x_{g}=R\right)$, the ball is not yet deformed and both the center of mass and the center of the sphere are at the same location. For larger and larger indentations the two centres separate one from another. Considering an homogeneous mass distribution of the membrane, the position of the center of mass during the contact reads

$$
x_{g}=R\left(1-\frac{x}{2 R}\right)^{2} \text {. }
$$

\section{Gas-membrane interaction during an impact}

\subsection{Material and methods}

The behaviour of the gas and its interaction with the membrane during an impact has been explored experimentally. In order to assess the hypothesis that the gas compression-expansion is adiabatic, one has to show that the time scale of thermal exchanges is larger than the impact time scale. To this aim, a first static compression experiment is performed to measure the characteristic time of thermal leaks. Second, the gas dynamical behaviour is analysed during the impact to understand how gas and membrane interact.

For both experiments, the membrane was punched with two holes of $5 \mathrm{~mm}$ of diameter. In each hole, a home-made hermetic feedthrough was inserted. The two feedthroughs allowed to place a micro-thermocouple and two pressure sensors inside the membrane simultaneously, see figure $3 \mathrm{c}$. The total mass of the feedthroughs and sensors was $6 \mathrm{~g}$, much lower than the membrane mass of $179 \mathrm{~g}(3.2 \%$ of the total mass). 

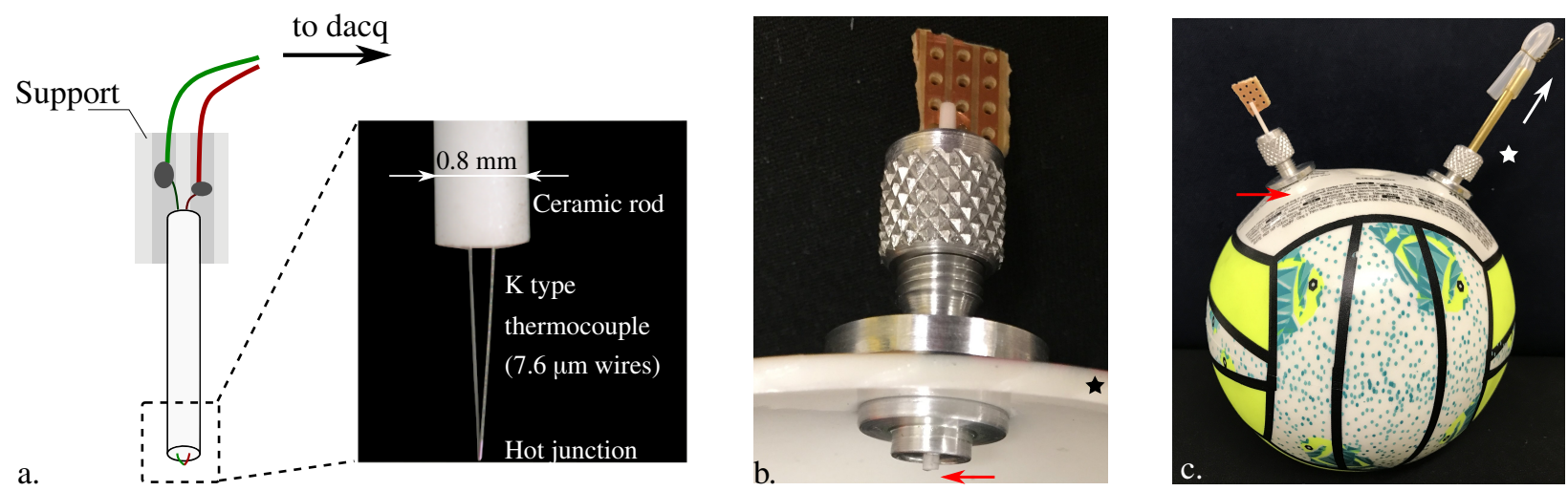

Figure 3: Instrumented membrane for gas pressure and temperature measurement. a. Building of a fast K-type thermocouple. b. Cross section of the membrane showing the insertion of the thermocouple in the hermetic feedthrough. Red arrow: thermocouple tip. Black star: membrane. c. Example of an instrumented mini-membrane (radius 57.5 $\mathrm{mm}$ ) with two feedthroughs. One is devoted to temperature measurement (red arrow) with the micro-thermocouple tip inside the membrane and the other is devoted to the absolute and differential pressure measurements (white star) with the white arrow indicating that the pressure sensors are outside the membrane.

The first pressure sensor, sensor A, was an absolute pressure sensor (Freescale semiconductor MPXAZ6115A, probing with an accuracy of $3.45 \mathrm{kPa}$ ), used to measure the initial pressure inside the membrane. The second sensor, sensor B, was a differential sensor (Freescale semiconductor MPXV7007) with accuracy of $500 \mathrm{~Pa}$. This sensor was used to measure with accuracy the variations of pressure during impacts and static compression experiment. Differential pressure measurements were corrected by $1 \mathrm{~ms}$, according to the sensor response time. Both sensors were linked to the same feedthrough. A manual valve allowed to set the reference of pressure for sensor B.

The indentation of the membrane was optically measured with a high-speed camera (Photron MIRO 110). The images were processed with a home-made Matlab ${ }^{\circledR}$ code similar to the one described in Section 2.1.

The temperature of the gas was recorded by a fast response type $\mathrm{K}$ thermocouple made by the welding of thin alumel and chromel wires (figure $3 a, b)$. The welded hot junction is realised by a capacitance discharge device developed by the FEMTO-ST laboratory [23]. The thin wires were insulated from each other by a ceramic rod of diameter $0.8 \mathrm{~mm}$. For the impact experiment, the use of extremely thin wires $(7.6 \mu \mathrm{m}$ in diameter) was required to get a sufficiently small thermal 
inertia to respond to sudden changes of temperature. The thermocouple of $7.6 \mu \mathrm{m}$ wires had a time constant of $\simeq 5 \mathrm{~ms}$ in air (natural convection) [23]. Conversely such a small thermocouple is mechanically fragile. To avoid breakage, a K thermocouple with wires of $75 \mu \mathrm{m}$ in diameter was used for the static compression experiment (time constant of $\simeq 1 \mathrm{~s}$ ). The home-made thermocouple was placed in the gas close to the membrane, figure $3 \mathrm{~b}$. Temperature signals were filtered for frequency content larger than $500 \mathrm{~Hz}$ and between 49.5 and $50.5 \mathrm{~Hz}$.

Tension signals were converted in absolute temperature using the tabulated Seebeck coefficient [24]. Sensitivity of the type $\mathrm{K}$ thermocouple was $40 \mu \mathrm{V} \mathrm{K}^{-1}$ at $293 \mathrm{~K}$ leading to an uncertainty of $\pm 0.2 \mathrm{~K}$ in our experiments. The main source of error was the noise due to antenna effect of the thermocouples.

All sensors were linked to a NI instruments dacq for acquiring the signal through a Labview interface. Data were acquired at $10^{4} \mathrm{~Hz}$. During impact experiments, the high-speed camera was synchronised with the other measurements (pressures and temperature) with a trigger signal.

\subsection{Experimental results}

The membrane has been statically compressed between two plans. The inner pressure rose quickly, figure $4 \mathrm{a}$, and was maintained for more than $80 \mathrm{~s}$ before being released. The rise of pressure induced an increase of the gas temperature, see figure $4 \mathrm{~b}$. The delay was due to the response time $(\simeq 1 \mathrm{~s})$ of the $75 \mu \mathrm{m}$ thermocouple. After reaching a maximum the temperature decreased following a damped exponential (straight line when plotted in lin-log scale, see inset). The characteristic time of decrease of temperature was $\tau_{\text {th }}=13.5 \mathrm{~s}$.

As the gas cooling is not uniform within the membrane, the characteristic time may depend on the position of the thermocouple relative to the membrane. The measured time $\tau_{\text {th }}=13.5 \mathrm{~s}$ compares well with a characteristic diffusive time $\tau_{t h} \sim l^{2} / D \simeq 5 \mathrm{~s}$ where $l=1 \mathrm{~cm}$ is the distance of the thermocouple to the membrane and $D=20 \times 10^{-6} \mathrm{~m}^{2} \mathrm{~s}^{-1}$ is the thermal diffusion coefficient in air. To get rid off this effect, one can compute an upper bound for thermal transfer by conduction, considering that the gas cools down to the membrane temperature over a thickness $\sqrt{D t_{c}}$

$$
\frac{\delta \mathcal{E}_{\mathrm{th}}}{\mathcal{E}_{\mathrm{th}}}=\frac{3 \sqrt{D t_{c}}}{R} .
$$



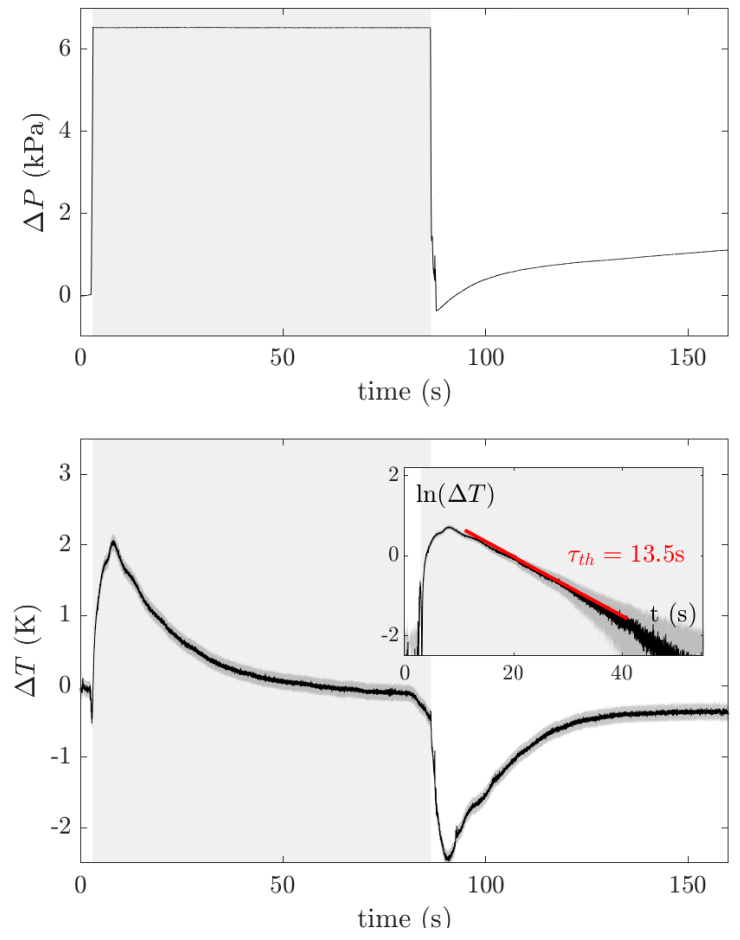

Figure 4: Simultaneous evolution of a. gas pressure variations $\Delta P$ and b. temperature variations $\Delta T$ for a long ( $>80$ s) compression-expansion cycle. (inset) lin-log graph of the gas temperature decrease showing the typical time $\tau_{t h}=13.5 \mathrm{~s}$. 

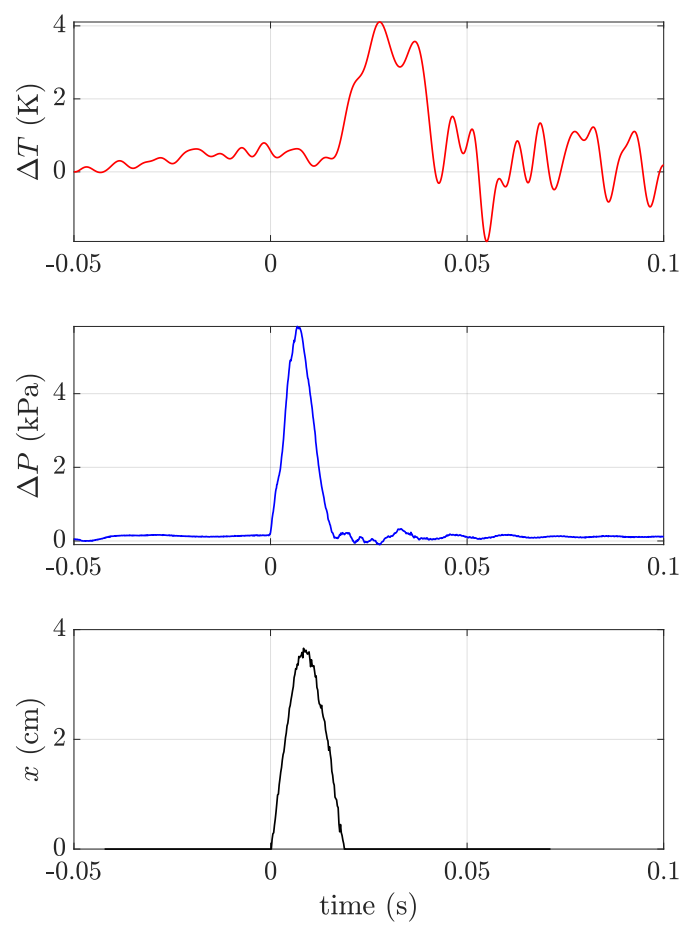

Figure 5: Simultaneous measurements of temperature variations $\Delta T$, pressure variations $\Delta P$ and indentation $x$ (for impact conditions $P=15.5 \mathrm{kPa}$ and $U_{0} \simeq 6 \mathrm{~m} \mathrm{~s}^{-1}$ ). Temperature sensor responds to temperature variations with a delay and has a moderate noise level.

Using typical values, $D=20 \times 10^{-6} \mathrm{~m}^{2} \mathrm{~s}^{-1}, t_{c}=10 \mathrm{~ms}$ and $R=10 \mathrm{~cm}$, one obtains $\delta \mathcal{E}_{\text {th }} / \mathcal{E}_{\text {th }} \simeq 1.3 \%$.

The adiabatic hypothesis is well verified.

The temperature variations, pressure variations and indentation were measured simultaneously for impacts at different heights $h$. A typical measurement is shown in Figure 5. The pressure and indentation evolved simultaneously due to the fast response of the sensors. Differently, the temperature only increased after a delay of $\simeq 5 \mathrm{~ms}$ corresponding to the response time of the $7.6 \mu \mathrm{m}$ thermocouple used in the experiment. The temperature increase shows that even for small indentation, gas compression is not isothermal and that gas temperature rises by few Kelvins. 


\subsection{Model of evolution of pressure during an impact}

The pressurised membrane was released at different heights $0.5 \mathrm{~m}<h<1.85 \mathrm{~m}$. The impact speed was much lower than sound velocity $\left(U_{0} \ll 340 \mathrm{~m} \mathrm{~s}^{-1}\right)$, it thus remained under the hypothesis of quasi-static compression. As shown previously, the gas compression was also adiabatic, it can be modelled by the Laplace relation,

$$
\frac{\Delta P}{\Delta V}=-\gamma \frac{P}{V},
$$

where $\gamma$ is the Laplace coefficient. $\gamma=5 / 3$ for monoatomic gases, $7 / 5$ for diatomic gases and $\gamma \rightarrow 1$ for polyatomic gases. During the impact, the membrane is an indented sphere which radius slightly increases because of pressure. The increase of radius of a thin elastic sphere reads [25],

$$
R=R_{0}+\frac{\left(P-P_{\mathrm{atm}}\right) R_{0}^{2}}{2 e E} .
$$

The dependence between membrane radius and inflation pressure is shown in figure 5a and fitted with $E=4.2 \mathrm{MPa}$ and $e=2 \mathrm{~mm}$ to determine $R_{0}=8.2 \mathrm{~cm}$. In the limit of small indentations $x \ll R$, one may linearise the membrane indented volume Eq (1), and the two above relations, Eqs. (4) and (5) to find the relation between gas pressure and indentation,

$$
\frac{\Delta P}{P}=\frac{\gamma}{1+(2+3 \gamma) P_{E}+(1+3 \gamma) P_{E}^{2}}\left(\frac{x}{R_{0}}\right)^{2} \quad \text { with } \quad P_{E}=\frac{P R_{0}}{2 E e} .
$$

$P_{E}$ is the presso-elastic number which scales the inflation pressure to the elasticity of the membrane. For $P_{E} \ll 1$, the membrane is rigid and the increase of radius is negligible. In the contrary if $P_{E} \gg 1$, the membrane is flexible, the membrane radius increases and pressure variations are reduced. In the case of the membrane studied experimentally $P_{E}$ is in the medium range with $P_{E} \simeq 0.56$ (using values in Table 1). The relation between pressure and indentation is plotted in Figure $5 \mathrm{~b}$ for all impact experiments $(0.5 \mathrm{~m}<h<1.85 \mathrm{~m})$, and compares well with the theory without fitting parameters. In the inset, the compression-expansion cycle is shown to occur without hysteresis. 


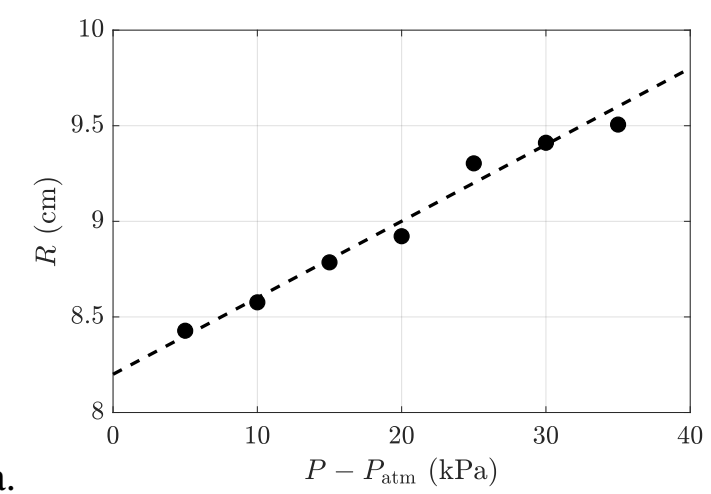

a.

$$
P-P_{\text {atm }}(\mathrm{kPa})
$$

b.

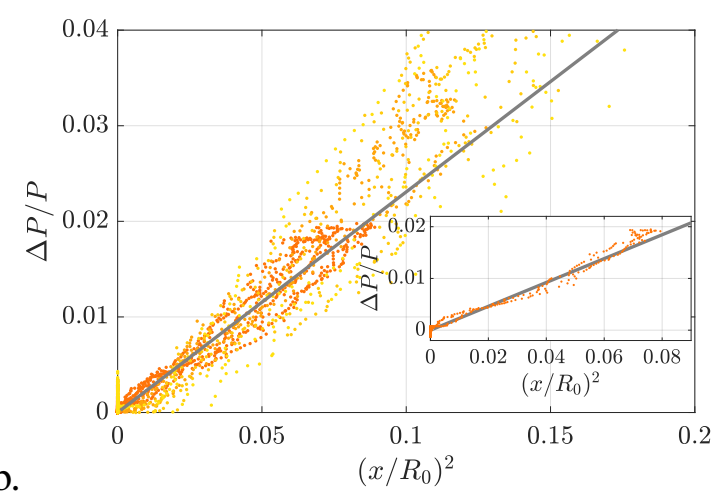

Figure 6: a. Membrane radius vs Inflation pressure. Colours correspond to impact speed following colour map of figure 1. Black line corresponds to Eq. (5) with $E=4.2 \mathrm{MPa}, e=2 \mathrm{~mm}$ and $R_{0}=8.2 \mathrm{~cm}$. b. Example of normalised pressure vs. normalised indentation squared for an impact from height $h=0.5 \mathrm{~m}$. Black line corresponds to Eq. (5) with $P_{E}=0.56$ and $\gamma=7 / 5\left(P=114 \mathrm{kPa}, E=4.2 \mathrm{MPa}, e=2 \mathrm{~mm}\right.$ and $\left.R_{0}=8.2 \mathrm{~cm}\right)$.

\section{Effect of gas nature and pressure on contact time}

\subsection{Conservation of momentum}

In the chosen parametrisation, we compute the acceleration of the center of mass as a function of $x$ by double differentiation of Eq. (2),

$$
\ddot{x}_{g}=-\left(1-\frac{x}{2 R}\right) \ddot{x}+\frac{\dot{x}^{2}}{2 R} .
$$

The right-hand-side of this equation is compounded of two terms: the first one corresponds to the variation of momentum due to the acceleration of the mass of the membrane that is not in contact with the ground, $m(1-x / 2 R)$. The second term corresponds to the variation of momentum associated to the membrane mass that actually reaches the ground during a given time interval, $m \dot{x} / 2 R$, which velocity drops from $\dot{x}$ to zero. When the membrane is not much squashed on the ground, $x \ll R$, the second term is much lower than the first one and the acceleration of the center of mass simply reduces to $\ddot{x}_{g}=-\ddot{x}(1-x / 2 R)$.

The force exerted by the membrane onto the ground is the inner pressure, $P+\Delta P(x)-P_{\mathrm{atm}}$ times the surface of contact, $A$. Conservation of momentum reads,

$$
m \ddot{x}_{g}=\beta A(x)\left(P+\Delta P-P_{\text {atm }}\right),
$$


where $\beta \simeq 1 / 2$ is the fraction of the flattened membrane in contact with the marble, see figure $1 \mathrm{~b}$. Under the hypothesis of small indentation $\dot{x}^{2} / 2 R \ll \ddot{x}$, one obtains

$$
-m \ddot{x}=2 \beta \pi R x\left(P-P_{\mathrm{atm}}\right)\left(1+\frac{\Delta P}{P-P_{\mathrm{atm}}}\right) .
$$

The variation of radius due to the increase of pressure is negligible compared to the variation of inner pressure during the impact as $\Delta R / R=R_{0} P_{E} \Delta P / P R<\Delta P / P \ll \Delta P /\left(P-P_{\text {atm }}\right)$. The quantity $\Delta P /\left(P-P_{\text {atm }}\right)$ which corresponds to the relative increase of pressure during the impact is thus the main source of force non-linearity in Eq (9). In the following, we neglect variations of membrane radius $R$. At this step, we define the dimensionless variables $\tilde{x}=x / R, \tilde{t}=t U_{0} / R$ and make Eq.(9) dimensionless. We obtain the presso-inertial number $I_{P}=m U_{0}^{2} / 2 \beta \pi R^{3}\left(P-P_{\mathrm{atm}}\right)$ and the reduced pressure $\tilde{P}=\left(P-P_{\text {atm }}\right) / P$. For convenience, we note $B=\gamma\left(1+P_{E}^{2}\right) /\left(1+(2+3 \gamma) P_{E}+(1+3 \gamma) P_{E}^{2}\right)$. Combining Eq. (9) and (6), one finds the dimensionless equation of evolution of the indentation,

$$
\ddot{\tilde{x}}=-\frac{\tilde{x}}{I_{P}}-\frac{B \tilde{x}^{3}}{I_{P} \tilde{P}}
$$

With the initial condition at $\tilde{t}=0, \tilde{x}=0$ and $\dot{\tilde{x}}=1$, the first integral of Eq. (9) reads

$$
\dot{\tilde{x}}= \pm \sqrt{1-\frac{1}{I_{P}}\left(\tilde{x}^{2}+\frac{B}{2 \tilde{P}} \tilde{x}^{4}\right)} .
$$

\subsection{Pressure, temperature and indentation maxima}

The maximal indentation occurs when $\dot{\tilde{x}}=0$. There exists a single physical solution that reads

$$
\frac{x_{\max }}{R}=\sqrt{\frac{\tilde{P}}{B}}\left(\sqrt{1+\frac{2 B I_{P}}{\tilde{P}}}-1\right)^{1 / 2} .
$$

Under the hypothesis of slow impact $I_{p} \ll 1$, one gets

$$
\frac{x_{\max }}{R}=\sqrt{I_{p}}\left(1-\frac{B I_{P}}{4 \tilde{P}}\right)+o\left(I_{P}^{3 / 2}\right) .
$$

Consequently, one finds the maximum of pressure and temperature elevations

$$
\frac{\Delta P_{\max }}{P}=B\left(\frac{x_{\max }}{R}\right)^{2} \text { and } \quad \frac{\Delta T_{\max }}{T}=\frac{(\gamma-1)}{\gamma} \frac{\Delta P_{\max }}{P} .
$$

The comparison of the predicted maxima to the experimental values is shown in Figure 7. The model predicts the right order of magnitude and tendency for indentation, pressure and temperature. 


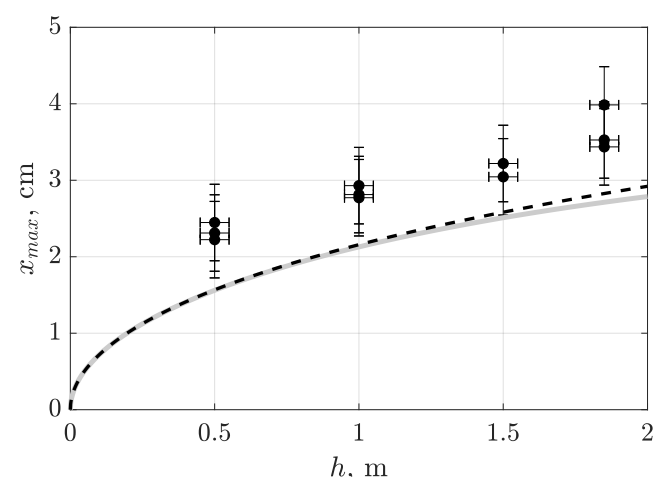

a.

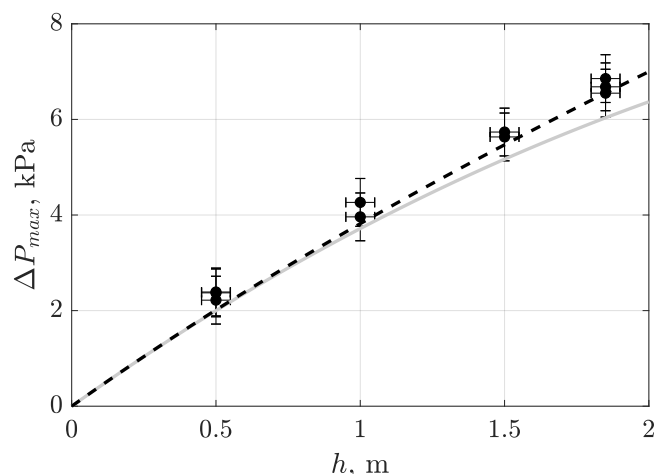

b.

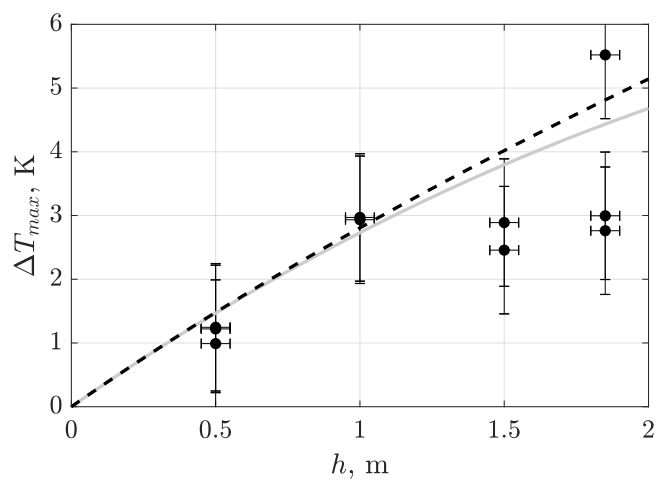

Figure 7: Maximal values of a. indentation $x, \mathrm{~b}$. pressure variation $\Delta P$ and c. temperature variation $\Delta T$, with release height of the membrane. Dashed lines correspond to the exact model prediction, Eq. (12) and (14) using $\gamma=7 / 5, P=114 \mathrm{kPa}, T=293.15 \mathrm{~K}$ and membrane characteristics given in Table 1. Light grey lines correspond to the approximated predictions, Eq. (13) and (14) with the same set of parameters. 


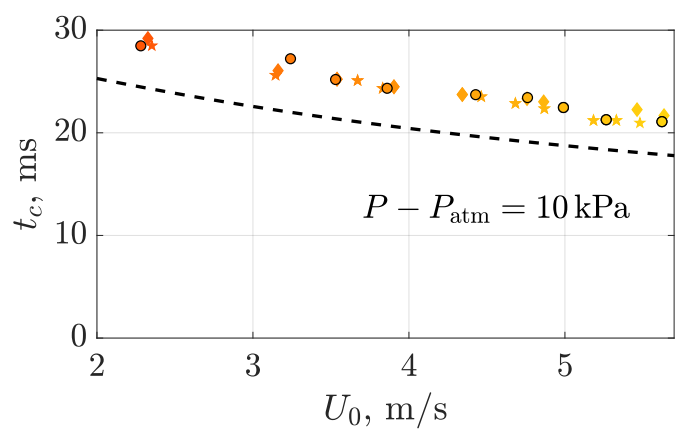

Figure 8: Comparison of contact time prediction and measurement for trial at $P-P_{\mathrm{atm}}=10 \mathrm{kPa}$. Black line: model prediction Eq.(15).

Since dissipation is not taken into account in this model, the contact time is twice the time needed to get to the maximal indentation,

$$
\left.t_{c}=\frac{2 R}{U_{0}} \int_{0}^{\tilde{x}_{\max }} \frac{\mathrm{d} \tilde{x}}{\sqrt{1-\frac{1}{I_{P}}\left(\tilde{x}^{2}+\frac{B}{2 \tilde{P}} \tilde{x}^{4}\right)}}=\frac{2 R}{U_{0}} \sqrt{\frac{2 I_{P}}{\sqrt{1+\frac{2 B I_{P}}{\tilde{P}}}+1}} \mathbf{K}-\frac{\sqrt{1+\frac{2 B I_{P}}{\tilde{P}}}-1}{\sqrt{1+\frac{2 B I_{P}}{\tilde{P}}}+1}\right) .
$$

where $\mathbf{K}$ is the first complete elliptic integral. We plot $t_{c}$ for various inflation pressure and velocities in Figure 2a. The model is in good agreement with the data trends. A discrepancy between model and data exists when comparing the magnitude of the contact time, figure 8. This discrepancy comes from the term in $\dot{x}^{2}$ in $\mathrm{Eq} 7$ that increases the contact time and from ball oval distortion.

\section{Discussion}

The model developed for the dynamics of a pressurised membrane impacting a rigid surface relies on several assumptions which should be discussed. First, we assumed that no energy was dissipated during the impact (neither sound emission [22], vibrations nor visco-elasticity dissipation $[21,26])$, that leads to overestimate the maximal indentation $x_{\max }$ and to underestimate the contact time $t_{c}$. This point will be addressed in a future study on coefficient of restitution, however it is thought to have little effect on the contact time $t_{c}$. Indeed, using a linear damped oscillator model, one may estimate the lost energy as $2 m \xi U_{0}^{2}$ where $\xi$ is the reduced damping coefficient. This compares to the lost energy during the impact $\left(1-\eta^{2}\right) m U_{0}^{2} / 2$. One finds $\xi \simeq\left(1-\eta^{2}\right) / 4$. 
The reduced damping coefficient changes the contact time as $t_{c, \text { damp }}=t_{c} / \sqrt{1-\xi^{2}}$. For $\eta=0.9$, $\left(t_{c, \text { damp }}-t_{c}\right) / t_{c} \simeq 10^{-3}$.

Second, we made the hypothesis of small indentations of the membrane $(x / R \ll 1)$ and consequently neglected the term $\dot{x}^{2} / 2 R$ in equation (7), this may induce an underestimation of the contact time. This term do not involve any loss of energy. Finally, we have accounted for oval distortion that arise because of membrane inertia through a simple corrective factor $\beta$. This is a raw assumption and taking into account the deformation of the ball due to inertia would be the next step of this theory. The quasi-static assumption is quite well verified in the limit of slow impact but may not be exact for impact at large velocities (approaching sound velocity or wave velocity in the membrane), possibly changing the physics of the impact.

Under these assumptions, we showed that the impact of a pressurised membrane is ruled by four dimensionless numbers. (i) $\tilde{P}$ is the normalised overpressure inside the membrane; (ii) the presso-elastic number $P_{E}$ compares the inflation pressure with the membrane elasticity; (iii) $I_{P}$ scales the initial kinetic energy with the work of the inner gas compression during the contact; finally, (iv) $\gamma$ is the Laplace coefficient that depends on gas nature. The first three parameters have a major influence of the contact dynamics. In the contrary, $\gamma$ is bounded between $5 / 3$ for the adiabatic compression of a mono-atomic gas and 1 for an isothermal compression. In this range of variations $(40 \%)$ it has only little influence on the contact time. The physical properties of the inflation gas (mono-atomic, polyatomic, etc...) only change marginally the bouncing dynamics of the membrane. Differently, the increase of the inner pressure implies an increase of $\tilde{P}$ and $P_{E}$ and provokes a reduction of the maximal indentation and of the contact time. When the impact speed is increased keeping the overpressure $\tilde{P}$ constant, $I_{P}$ becomes larger and induces a larger maximal indentation and a shorter contact time.

In order to compare the model predictions with experiments, we characterised the impact dynamics of a model pressurised membrane onto a rigid substrate for different inflation pressures and impacting speeds. These experiments lied on the range of parameters where $P_{E}<1$ and $I_{P}<1$, corresponding to small membrane elasticity and slow impacts. The good agreement between these observations and model predictions justify the approximations discussed previously. One perspective of this work would be to change the properties of the membrane in order to explore the limit 
where the non-dimensional numbers $P_{E}$ and $I_{P}$ are larger than unity and verify model predictions in this limit.

This model of contact time of pressurised membranes allows to discuss the properties of inflated protections and sport balls. Biological damages are difficult to relate to a unique impact property as, for instance, traumatic brain injuries causes and criteria are numerous and complex [27]. Nevertheless, biological damages are somehow dependent of the maximum force level. In the case of a free pressurised membrane the maximum force is related to the contact duration as $F_{\max } \sim m U / t_{c}$. Increasing contact time by decreasing inflation pressure or by choosing softer membrane may thus allow to reduce impact injuries. This should help to design safer sports balls [3]

In this paper, we considered an ideal target of infinite mass and stiffness. This may represent quite well the impact of a pressurised sport ball on a hard ground such concrete or wooden floor that may be encountered in basketball or handball. Differently, for outdoor sports such as football or rugby, the field is soft enough to be deformed elastically or plastically by the ball during the impact, changing the properties of impact $[28,29]$. This remark stands also for impact of pressurized balls or pressurised protections on humans which soft tissues (skin, muscles, etc.) may deform under impact. To take into account the mass of the target one has to consider the relative velocities in the frame of the center of mass. Compared to the case of infinite target mass $m_{t} \rightarrow \infty$, the amount of momentum exchanged during the impact is reduced by a factor $m_{t} /\left(m_{i}+m_{t}\right)$ where $m_{i}$ is the mass of the impactor. Regarding the stiffness, the springs of the target $k_{t}$ and impactor $k_{i}$ are in series what decreases the total stiffness of the system $k_{i} k_{t} /\left(k_{i}+k_{t}\right)$ and increases contact time consequently. Thus taking into account a real target decreases the amount of momentum exchanged and increases contact time. The case of an impact on an ideal target represents thus an upper limit for inertial forces.

Regarding sports, it is unlikely that increasing contact time may increase precision since human response time is much larger than ball contact time. Differently, longer contact time may favour handling and control of the ball since it mostly relies on motion coordination rather than reflex. This explain why ball deflation has been used to cheat in American football where each team uses its own ball, [30]. Questions also arise for the level of trajectory modification following the 
impact of a spinning ball depending on its inflation pressure since it will increase indentation, contact surface and contact time.

\section{Conclusion}

We have shown that the contact dynamics of a pressurised membrane is made non-linear by the increase of the internal pressure resulting from the adiabatic compression of the gas. We have proposed a non-linear model that rationalizes the dependency of the contact time with the inflation pressure and the impacting speed. In addition, this model predicts a small effect of the gas nature, a fact that is observed in practice. Our model implies that the contact force $F$ depends on the indentation $x$ in a supra-linear way: $F \propto \tilde{x}+B \tilde{x}^{3} / \tilde{P}$. The bouncing dynamics of a pressurised membrane strongly differs from the Hertzian law $F \propto x^{3 / 2}$ which rules the impact of filled spheres made of elastic materials. It is also different from the linear law for shells before buckling $F \propto x$ if $x<e$ and the sub-linear law for shells after buckling $F \propto x^{1 / 2}$ for $x>e$ [31]. It would be interesting to study the implications of such differences in sports where these distinct types of balls are employed.

\section{Acknowledgement}

The authors thanks Stéphane Méo and Marie-Pierre Deffargues for their welcome at CERMEL lab and use of the traction bench. The authors thanks Laurent Aprin and Tristan Gilet for the loan of high-speed cameras. Authors thanks Tristan Gilet, Christophe Clanet, Caroline Cohen, Aisha Medina, Jean-Marc Linares, Santiago Arroyave-Tobòn and Julien Chaves-Jacob for valuable help and/or discussions.

\section{References}

[1] J. Heald, D. Pass, Ball standards relevant to risk of head injury, in: Head and neck injuries in sports, ASTM International, 1994.

[2] B. C. Buckhout, M. A. Warner, Digital perfusion of handball players: effects of repeated ball impact on structures of the hand, The American journal of sports medicine 8 (1980) 206-207. 
[3] N. J. Cecchi, D. C. Monroe, W. X. Moscoso, J. W. Hicks, D. J. Reinkensmeyer, Effects of soccer ball inflation pressure and velocity on peak linear and rotational accelerations of ball-to-head impacts, Sports Engineering 23 (2020) 1-6.

[4] S. Pang, W. Goldsmith, A model of a pneumatic jackhammer system, Rock mechanics and rock engineering 25 (1992) 49-61.

[5] N. Mills, Running shoe materials, Materials in sports equipment 1 (2003) 65.

[6] M. Kurt, K. Laksari, C. Kuo, G. A. Grant, D. B. Camarillo, Modeling and optimization of airbag helmets for preventing head injuries in bicycling, Annals of biomedical engineering 45 (2017) 1148-1160.

[7] H. Zhou, Z. Zhong, M. Hu, Design and occupant-protection performance analysis of a new tubular driver airbag, Engineering 4 (2018) 291-297.

[8] A. H. ye, Are airbags a dangerous safety measure? a meta-analysis of the effects of frontal airbags on driver fatalities, Accident Analysis \& Prevention 42 (2010) 2030 - 2040. doi:https://doi.org/10.1016/j.aap.2010.06.014.

[9] T. Koisaari, T. Leivo, A. Sahraravand, A.-K. Haavisto, P. Sulander, T. M. Tervo, Airbag deployment-related eye injuries, Traffic injury prevention 18 (2017) 493-499.

[10] E. Falcon, C. Laroche, S. Fauve, C. Coste, Behavior of one inelastic ball bouncing repeatedly off the ground, The European Physical Journal B-Condensed Matter and Complex Systems 3 (1998) 45-57.

[11] J. C. Bird, R. Dhiman, H.-M. Kwon, K. K. Varanasi, Reducing the contact time of a bouncing drop, Nature 503 (2013) 385-388

[12] D. Richard, C. Clanet, D. Quéré, Contact time of a bouncing drop, Nature 417 (2002) 811-811.

[13] F. Vincent, A. Le Goff, G. Lagubeau, D. Quéré, Bouncing bubbles, Journal of Adhesion 83 (2007) 897-906.

[14] A. Lazarus, H. Florijn, P. M. Reis, Geometry-induced rigidity in nonspherical pressurized elastic shells, Physical review letters 109 (2012) 144301.

[15] D. Vella, A. Ajdari, A. Vaziri, A. Boudaoud, The indentation of pressurized elastic shells: from polymeric capsules to yeast cells, Journal of the Royal Society Interface 9 (2012) 448-455.

[16] L. Pauchard, S. Rica, Contact and compression of elastic spherical shells: the physics of a ping-pong ball, Philosophical Magazine B 78 (1998) 225-233.

[17] M. Hubbard, W. J. Stronge, Bounce of hollow balls on flat surfaces, Sports Engineering 4 (2001) 49-61.

[18] W. Stronge, A. Ashcroft, Oblique impact of inflated balls at large deflections, International journal of impact engineering 34 (2007) 1003-1019.

[19] R. Cross, Dynamic properties of tennis balls, Sports Engineering 2 (1999) $23-34$.

[20] R. Cross, Impact behavior of hollow balls, American Journal of Physics 82 (2014) 189-195.

[21] A. Georgallas, G. Landry, The coefficient of restitution of pressurized balls: a mechanistic model, Canadian Journal of Physics 94 (2015) 42-46.

[22] J. Katz, Thump, ring: the sound of a bouncing ball, European journal of physics 31 (2010) 849. 
[23] F. Lanzetta, E. Gavignet, Temperature measurements: Thermoelectricity and microthermocouples, Thermal Measurements and Inverse Techniques (2011) 95.

[24] E. Rathakrishnan, Instrumentation, measurements, and experiments in fluids, CRC Press, 2007.

[25] J. Sigüenza, S. Mendez, D. Ambard, F. Dubois, F. Jourdan, R. Mozul, F. Nicoud, Validation of an immersed thick boundary method for simulating fluid-structure interactions of deformable membranes, Journal of Computational Physics 322 (2016) 723-746.

[26] A. Ashcroft, W. Stronge, Dynamic dissipation during bounce of tennis balls, The Engineering of Sport 1 (2004) 386-392.

[27] J. Antona-Makoshi, Traumatic brain injuries: animal experiments and numerical simulations to support the development of a brain injury criterion, Chalmers University of Technology Gothenburg, Sweden, 2016.

[28] M. Ali, J. Sun, N. Lam, L. Zhang, E. Gad, Simple hand calculation method for estimating deflection generated by the low velocity impact of a solid object, Australian Journal of Structural Engineering 15 (2014) 243-259.

[29] Y. Yang, N. Lam, L. Zhang, Estimation of response of plate structure subject to low veloctiy impact by a solid object, International Journal of Structural Stability and Dynamics 12 (2012) 1250053.

[30] K. A. Hassett, J. W. Sullivan, S. Veuger, Football under pressure: Assessing malfeasance in deflategate, Journal of Sports Analytics 1 (2015) 103-110.

[31] B. Audoly, Y. Pomeau, Elasticity and geometry: from hair curls to the non-linear response of shells, Oxford university press, 2010. 